\title{
Life history, cognition and the evolution of complex foraging niches
}

Caroline Schuppli ${ }^{\mathrm{a} *}$, Sereina M. Graber ${ }^{\mathrm{a}}$, Karin Isler, Carel P. van Schaik

Anthropological Institute and Museum, University of Zurich, Winterthurerstrasse 190, CH-8057 Zurich, Switzerland

ajoint first author

*Corresponding author

E-mail addresses: caroline.schuppli@aim.uzh.ch (C. Schuppli), sereina.graber@uzh.ch (S. M. Graber), karin.isler@biol.uzh.ch (K. Isler), vschaik@aim.uzh.ch (C.P.van Schaik)

\begin{abstract}
Animal species that live in complex foraging niches have, in general, improved access to energy-rich and seasonally stable food sources. Because human food procurement is uniquely complex, we ask here which conditions may have allowed species to evolve into such complex foraging niches, and also how niche complexity is related to relative brain size. To do so, we divided niche complexity into a knowledge-learning and a motor-learning dimension. Using a sample of 78 primate and 65 carnivoran species, we found that two life-history features are consistently correlated with complex niches: slow, conservative development or provisioning of offspring over extended periods of time. Both act to buffer low energy yields during periods of learning, and may thus act as limiting factors for the evolution of complex niches. Our results further showed that the knowledge and motor dimensions of niche complexity were correlated with pace of development in primates only, and with the length of provisioning in only carnivorans. Accordingly, in primates, but not carnivorans, living in a complex foraging niche requires enhanced cognitive abilities, i.e. a large brain. The patterns in these two groups of mammals show that selection favors evolution into complex niches (in either the knowledge or motor dimension) in species that either develop more slowly or provision their young for an extended period of time. These findings help to explain how humans constructed by far the most complex niche: our ancestors managed to combine slow development (as in other primates) with systematic provisioning of immatures and even adults (as in carnivorans). This study also provides strong support for the importance of ecological factors in brain size evolution.
\end{abstract}

Keywords: niche complexity, skill learning, food processing, development, provisioning, brain size

\section{Introduction}

There is extensive variation in the foraging niches different mammal species occupy. Whereas some foraging niches seem to be simple because they involve no pre-ingestive processing (e.g. those occupied by grazing ungulates), others appear to be more complex, because obtaining access to food requires multiple processing steps, executed in the correct order and timed properly (as occupied by many primates [e.g. Gibson, 1986; Byrne et al., 1993; Gunst et al., 2010]). Living in a complex foraging niche may bring palpable fitness benefits (Gibson, 1986). First, foods that require a high level of processing, such as underground storage organs, insects or other animal prey consistently show a high nutritive content. Second, because extracted foods are often available year-round, species able to exploit them can live in seasonal environments in which they would otherwise 
experience a lean season. Understanding the evolution of complex niches is important for human evolution because, unique among primates, human hunter-gatherers (as model for ancestral humans), and indeed humans in general, rely on highly complex forms of extractive foraging and hunting, and so manage to maintain a relatively stable energy intake in a great variety of different environments (Leonard and Robertson, 1997; Kaplan et al., 2000; Berbesque et al., 2014).

So far, no study has systematically examined the factors that allow species to evolve into such complex foraging niches. Occupying a complex foraging niche will generally require lengthy periods of learning, during which failure is common and net yields are low. Since these learning periods are costly we expect them to be connected to life history features that counterbalance these costs. Indeed, we recently found that species with a late age at skill competence (the age at which adultlevel skill levels are attained) are those that show one of two enabling factors: post-weaning provisioning or slow, conservative development (Schuppli et al., 2012). Both factors work as an energetic buffer against failures during periods of learning and therefore allow the learning period to be extended (Fig. 1). We also found evidence that species with complex foraging niches (with complexity defined as the level of processing required) reach adult-level feeding skills later in development than those that live in simpler niches (Schuppli et al., 2012).

In this paper, we ask whether foraging-niche complexity coevolved with long periods of learning or provisioning and with brain size. Such a three-way relationship has often been suggested by anthropologists, who proposed that slowly developing species do so because they need time to learn skills essential to sustain reproduction (Janson and van Schaik, 1993). A recent study in which this was quantified, however, showed that the time needed to learn these skills limited the duration of development only in a subset of species, including humans (Schuppli et al., 2012), and that the most widespread limiting factor is a tradeoff between energy allocation to needs of a growing body and a growing and differentiating brain. As a result, larger-brained species develop more slowly (Isler and van Schaik, 2009; Barton and Capellini, 2011) and thus reach maturity at a later age, which is compensated for by their improved adult survival (Isler and van Schaik, 2009; Gonzalez-Lagos, 2010). In humans, this tradeoff is responsible for our highly delayed maturation and the adolescent growth spurt (Kuzawa et al., 2014), although in humans adult-level skills are reached even later (Kaplan et al., 2000).

Nonetheless, there are good reasons to assume there is a link between niche complexity and brain size. First, larger brains are found in species with higher overall diet quality (Fish and Lockwood, 2003) or those that engage in extensive extractive foraging or tool use (Byrne, 1997; Reader and Laland, 2002; Barton, 2012). Second, larger brains are found in species that can maintain a high and stable energy intake all year round (van Woerden et al., 2010, 2012, 2014), often as a result of extractive foraging techniques (Gibson, 1986) or perhaps because of the ability locate ephemeral food sources (Milton, 1988). This same argument has also been applied to human evolution. It has repeatedly been suggested that the need to invent complex foraging techniques in an increasingly seasonal habitat was a driving force in the evolution of human intelligence (e.g. Parker and Gibson, 1977; Byrne, 1997; Anton et al., 2014). However, so far only very few studies have looked at the relation between brain size and niche complexity, and the ones that have focused on a few taxa only and produced inconsistent results (Parker and Gibson, 1977; Milton, 1981; Gibson, 1986; Walker et al., 2006). 
Here, we first examine whether the two factors that allow for extended periods of learning during development (slow development and post-weaning provisioning) are also a prerequisite for evolving into a more complex foraging niche (Fig. 1). Focusing on the direct link between niche complexity and provisioning and pace of development allows us to include a much broader sample of species than in the previous study (Schuppli et al., 2012) where limited data on age of skill competence led to small sample size. Second, in order to attain a better understanding of the cognitive aspect of niche complexity, we examine how different aspects of niche complexity relate to brain size. If we find a relationship between foraging-niche complexity and relative brain size across different species, this may help to explain why species with complex foraging niches are relatively rare and why humans occupy by far the most complex niche.

A key decision in a study of foraging-niche complexity is how to define complexity. Previous studies have ranked the skill requirements of different food types and consistently classified leaves and grasses as less skill intensive than items, such as fruit, that require some kind of manipulation with hands or coordinated movements involving both hands and parts of the mouth (teeth, lips). The ingestion of embedded food items, such as nuts, which require more processing steps is generally considered to require more complex skills (Dittus, 1977; Kaplan et al., 2000; Johnson and Bock, 2004). All these studies thus used the amount of processing with hands or hands and mouth required as a measure of complexity, such that items that need few or no processing steps are rated as less skill-intensive than items that require a feeding technique composed of several steps of processing. Other studies have classified specific elements of the diet or certain processing techniques, such as tool use, extractive foraging or cooperative hunting, as complex since they are based on knowledge and their efficiency improves with causal understanding (Holekamp et al., 1997; Gurven et al., 2006; Lonsdorf, 2006; Gunst et al., 2010).

The patterns found in these studies suggest that ecological niche complexity can be divided in two broad dimensions: knowledge and motor complexity. Knowledge-niche complexity comprises knowing what to eat, where to look for it (which is not always obvious with embedded foods), which processing techniques to use, and how to integrate these techniques into an ordered sequence (cf. Barton, 2012). Since acquiring the requisite knowledge and understanding requires a learning period, we expect to find that species inhabiting complex knowledge niches show a long period of provisioning and/or a slow development. Motor-niche complexity, in contrast, encompasses the motor patterns involved in food acquisition and is consequently determined by the motor skills needed in each single step of food processing. We expect motor-niche complexity to be based more on practice than cognitive understanding. Unfortunately, it will be difficult to clearly distinguish between the two complexity dimensions in all cases since there may be substantial overlap between them.

In this study, we systematically test on a large comparative data set how foraging-niche complexity is related to the two life-history pathways, pace of development and the length of provisioning (Part I), and then, to relative brain size (Part II). We do this in two mammalian lineages: primates and carnivorans. For the life-history pathways, we expect that the pace of development or the length of provisioning underwent correlated evolution with niche complexity, such that species living in more complex foraging niches are characterized by slower development or extended provisioning. We are also interested in how strongly each of the two different dimensions of niche complexity (knowledge and motor) is correlated with development or provisioning. For the link between foraging niche complexity and cognition, we expect that knowledge-niche complexity (comprising knowledge and 
understanding of a foraging niche) is positively correlated with relative brain size. However, the prediction for the relation between brain size and motor niche is less straightforward. On the one hand, we assume the motor dimension to require extensive practice, but not necessarily understanding and knowledge, and therefore not to rely on enhanced cognition. On the other hand, practice itself as well as sensory-motor processes involved in motor skills are expected to have neural correlates, which would suggest a positive correlation between brain size and motor-niche complexity. In other words, we hypothesize a large brain to be an inevitable prerequisite for species living in a complex knowledge niche but not necessarily for those living in a complex motor niche.

\section{Methods}

\section{Data sets}

We compiled data sets on the different elements of niche complexity, life history parameters, brainand body size for 78 nonhuman primate and 65 carnivoran species. Life history, provisioning, brain and body size data were retrieved from established databases (K. Isler, S. M. Graber and S. Heldstab, unpublished data; Isler and van Schaik, 2009, 2012; van Woerden et al., 2014). Data on the different elements of niche complexity were taken from the published literature (see Supplementary Online Material [SOM]). We use measurements on overall brain size as this has been shown to be a very good predictor of cognitive abilities (Lefebvre et al., 2004; Deaner et al., 2007) and is a very broadly available measurement in mammals.

For motor-niche complexity, we divided all species into three complexity categories according to the level of processing involved in food acquisition (Table 1). For grazing and folivory, food acquisition generally comprises only one step (bite), whereas for frugivory and insectivory, two basic steps are almost always required (e.g. pick and bite, or peel and bite, or bite and spit), and for extractive foraging and catching mobile prey, three or more basic steps are required (e.g. extract and bite and spit, or catch and kill and bite). As expected, this complexity ranking roughly has a cumulative (Guttman) scaling property, such that species in category 2 or 3 mostly also exhibit elements of category 1 , or 1 and 2, respectively.

For knowledge-niche complexity we determined four different cognitively challenging elements found in primates and carnivorans: cooperative hunting, high diet breadth, extractive foraging and tool use. These elements were coded as binary variables, except for diet breadth, where we counted how many different classes of food types a species eats. We also compiled a knowledge-niche complexity score by summing the number of cognitively challenging elements for a certain species, assuming that the more knowledge elements there are, the more complex the overall knowledge niche should be. For the knowledge score we dichotomized diet breadth into a binary variable by assigning $1-2$ different classes of food types to 0 (low), and 3 and more different food types to 1 (high). In primates, the observed additive knowledge-niche complexity scores ranged from 0 to 4 , whereas in carnivorans they ranged from 0 to 3 (Table 2).

\section{Variables}

To approximate the pace of development we used female age at first reproduction (AFR), controlled for female body mass through multiple regression analysis (see below). The relative length of provisioning was approximated by the total number of days offspring are provisioned, again corrected for body mass. Since there is no post-weaning provisioning in most primate species, we 
took weaning age as a measure for the length of provisioning for all species except the cooperatively breeding marmosets (Callithrix jacchus).

\section{Analysis}

All analyses and plots were done using the R programming language ( $R$ development Core Team, 2011). More closely related species, in general, show greater similarities in traits because of their shared evolutionary history (Nunn, 2011). To correct for phylogenetic non-independence of the data we used phylogenetic least-squares (PGLS) in the case of multistate ordinal response variables (as justified by the results of Matthews et al., 2010), as implemented in the package caper (Orme, 2011), and phylogenetic logistic regressions (PLR) in the case of binary response variables, as implemented in the package phylolm (Ho and Ane, 2014). The analyses testing the effects on the two foraging niche dimensions were all controlled for body mass (by including it as a factor in the regression models) to ensure that the effects were independent of body mass. In other words, throughout this study we consider life-history traits and brain size relative to body mass. Residuals of the models, as shown in the graphs, were used for illustrative purposes only. We also controlled for additional potentially confounding variables and provided these analyses in the SOM (Tables S2, S3, S4).

In PGLS, the phylogenetic scaling parameter lambda ( $\lambda$ ) (Pagel, 1999), a measure of phylogenetic signal in the model residuals, was estimated based on maximum likelihood as implemented in caper, whereas the other two branch-length transformation factors, delta and kappa, were fixed at 1.

The PLR in phylolm automatically estimates alpha $(\alpha)$, the phylogenetic signal for a binary trait. In contrast to $\lambda, \alpha$ is based on a Markov process and larger values correspond to a weaker phylogenetic signal (Ives and Garland, 2010). In order to reach evenly distributed residuals around zero, all continuous variables were log-transformed. To assess statistical significance, a $5 \%$ level was used. The phylogenetic trees used for the analyses were retrieved and compiled from the published literature (Bininda-Emonds et al., 2008; Perelman et al., 2011). A nexus file was manually extracted from the information provided in Perelman et al. (2011) and is provided in the SOM.

\section{Data classification}

We used the published literature to assign species to the various niche categories. However, this was not always straightforward since different sources used different classifications. We therefore repeated our analyses using various classifications, but this did not change our results, which we therefore consider robust. The classification presented here is conservative and in most cases not in favor of our predictions (e.g. Gorilla gorilla was not classified as an extractive forager whereas Callithrix jacchus was). To further test the robustness of our results, we also dichotomized all our ordinal dependent variables since in most cases this led to more balanced data distributions. This too, did not change the results. Our complete data sets with all classifications and their sources, are provided in the SOM (Table S1).

\section{Results}

Part I - Life history pathways and foraging-niche complexity 
In primates we found a significant positive effect of the pace of development on motor-niche complexity but no significant effect of the length of provisioning (approximately weaning age; Table 3a; Fig. 2). For carnivorans, in contrast, we found a significant positive effect of the length of provisioning on motor-niche complexity but no significant effect of the pace of development (Table 3b; Fig. 2).

\section{Knowledge-niche complexity}

For the overall knowledge-niche complexity score (summed number of cognitively challenging elements) we found the same pattern as for motor-niche complexity: in primates, the knowledgeniche complexity score showed a significantly positive correlation with the pace of development, whereas in carnivorans it showed a significantly positive correlation with the length of provisioning (Table 4a+b; Fig. 3).

Examining the different elements of knowledge-niche complexity separately, we found that in primates the pace of development had a significantly positive effect on diet breadth, extractive foraging and tool use. Since chimpanzees are the only cooperatively hunting primate species in our data set, we could not evaluate the effect of cooperative hunting statistically, but following our expectations, chimpanzees have a relatively slow pace of development (SOM Table S2a; Fig. S1).

In carnivorans, the length of provisioning had a significant positive effect on diet breadth. For cooperative hunting, there was a weak trend in the predicted direction whereas for extractive foraging no effect was apparent. For tool use, the small sample size did not allow for statistical testing in carnivorans (SOM Table S2b; Fig. S2).

In primates we found that motor-niche complexity and the additive knowledge-niche complexity score were highly correlated (PGLS: $p<0.001, \lambda=0.50$ ). However, no such correlation was found in carnivorans (PGLS: $p=0.35, \lambda=0.16$ ). In order to exclude a spurious effect of pace of development or provisioning on the knowledge-niche complexity score in primates, we included motor-niche complexity as a factor into our analysis of the predictors of the knowledge-niche complexity. We found that the effect of the pace of development on the knowledge-niche complexity score still held when we controlled for motor-niche complexity (primates: P (log AFR) $=0.038, p$ (motor complexity) $<0.001, \lambda=0$; see SOM Table S2a). Detailed statistics on the regression models and further analyses are also shown in the SOM (Table $\mathrm{S} 2 \mathrm{a}+\mathrm{b})$.

\section{Part II - brain size and foraging-niche complexity}

\section{Motor-niche complexity}

In carnivorans, there is no significant correlation between motor-niche complexity and brain size (Table 5b, Fig. 4). Adding the length of provisioning as an additional factor did not change the result (SOM Table S3b). In primates, in contrast, this correlation is significant (Table 5a; Fig. 4). However, the correlation between brain size and motor-niche complexity disappears if we control for AFR (SOM Table S3a).

\section{Knowledge-niche complexity}

In primates, the additive knowledge-niche complexity score showed a significant positive correlation with brain size (Table 6a; Fig. 5). In fact, the PGLS regression continued to show a significant 
correlation between brain size and the knowledge-niche complexity score even after additionally controlling for possible confounding effects of motor-niche complexity or for AFR (SOM Table S4a). These results are consistent with our hypothesis that predicted that species living in complex knowledge niches have bigger brain sizes. When analyzing the different cognitively challenging elements separately and controlling for the motor-niche dimension, only tool use showed a significant association with relative brain size (SOM Table S4a, Fig. S3). Also, being the only cooperatively hunting primate species in our data set, chimpanzees have a relatively large brain.

For carnivorans, in contrast, no such link between knowledge-niche complexity and relative brain size was found. Neither the additive knowledge-niche complexity score, nor the regression models of the individual cognitive elements showed evidence for a correlation with brain size (Table 6b, Fig. 5; SOM Table S4b, Fig. S4). Detailed statistics of the regression models of all additional analyses are also shown in the SOM (Table S4a+b).

\section{Discussion}

\section{Part I - Life history pathways to complex foraging niches}

In the first part, we found that two life history features are consistently correlated with both dimensions of niche complexity: in primates, slow, conservative development, and in carnivorans, provisioning over extended periods of time. Because newly weaned mammals of species living in complex foraging niches generally have not yet reached adult-level feeding skills (Schuppli et al., 2012), both features may help to provide an energetic buffer during periods of learning. Energy deficits caused by failures (and therefore lower net energy intake) during the period of learning may have severe consequences for the still growing juvenile individual because they carry the risk of brain starvation (Janson et al., 1993; Isler and van Schaik, 2009; Kuzawa et al., 2014). Post-weaning provisioning suppresses these energy deficits not just because it provides the immature animal with energy but also because it reduces the immature animal's required foraging time, and consequently allows it to allocate more time to practicing. Similarly, slow, conservative development reduces the risk of brain starvation and provides the immature animal with a longer pre-reproductive period during which it can afford to have the reduced foraging efficiency caused by learning. Because reproduction would compete for energy with maintenance, it is postponed until adequate skill levels have been reached. Thus, both mechanisms provide species with enhanced opportunities to learn after weaning. In support of this interpretation, adult-level feeding skills are reached at a relatively later point during ontogeny in those species that get provisioned longer or show an overall slower development (Schuppli et al., 2012).

When we divided niche complexity into a knowledge and a motor dimension we found consistent patterns for both dimensions: a positive correlation with the length of provisioning in carnivorans, and a positive correlation with the pace of development in primates. In terms of motor complexity, this clear finding is in line with our initial prediction that complex motor patterns need to be practiced over extended periods of time. Species that live in complex motor niches often do not rely exclusively on food requiring complex motor patterns but also forage on less skill-intense food items. As one would expect, it has been observed that in these species the different skills ontogenetically develop in the order of their complexity level, as in Japanese monkeys (Macaca fuscata [Nakayama 
et al., 1999; Hanya, 2003]), or in the order of moving from small to large prey in carnivorans and primates (Watt, 1993; Holekamp et al., 1997; Stone, 2006). Furthermore, complex foraging elements seem to be practiced throughout ontogeny (e.g. beach hunting in the bottlenose dolphin, Tursiops sp. [Sargeant et al., 2005]).

In terms of knowledge-niche complexity, we found similar correlations for most of the a priori defined cognitively demanding elements (cooperative hunting, diet breadth, extractive foraging and tool use). This finding confirms our initial prediction that acquiring knowledge and understanding requires a long period of learning. In line with our finding, previous studies have shown that all cognitively demanding elements are mastered relatively late in development: tool use in great apes (Lonsdorf, 2006; Meulman and van Schaik, 2013), cooperative hunting in different carnivorans and chimpanzees (Holekamp et al., 1997; Boesch, 2002; Sand et al., 2006) and extractive foraging in various primates (Johnson and Bock, 2004; Gunst et al., 2008; Schuppli et al., 2012).

Knowledge- and motor-niche complexity inevitably overlap since executing one step in the chain of processing steps needed for food acquisition automatically also means being able to coordinate and time all the previous steps needed to get to this step, which has been proposed to explain correlations between brain size measures and tool use (Barton, 2012). Indeed, some of the cognitively demanding elements we used are based on complex, multistep motor patterns. This is especially true for extractive foraging, where it is hard to determine to what extent the observed positive correlation between developmental pace and extractive foraging in primates is based on complex motor patterns that need to be practiced over an extended period of time rather than on cognitive understanding and therefore time to learn. This argument predicts a correlation between motor complexity and the knowledge-niche complexity score, which was indeed found in primates. However, when corrected for motor complexity, we still found an independent effect of the pace of development on knowledge-niche complexity. In order to distinguish properly between the motor and knowledge complexity of foraging niches, we will use the relationship of the two dimensions of the foraging niche with brain size.

\section{Part II - brain size and foraging-niche complexity}

Consistent with our initial prediction, there was no relationship between brain size and the motor dimension of niche complexity in carnivorans. In primates, in contrast, brain size was positively correlated with motor-niche complexity, but after controlling for AFR, this correlation disappeared. Age at first reproduction has been shown to be associated with both brain size (Harvey and Clutton Brock, 1995; Ross and Jones, 1999; Barrickman et al., 2008) and niche complexity (see results Part I). One interpretation, therefore, is that the correlation between brain size and motor-niche complexity is spurious, because it is, in fact, driven by the duration of development (as in carnivorans, where is driven by the length of provisioning). Alternatively, an extended period of development is indeed the mechanism that allows for the development of a large brain (Barton and Capellini, 2011), which in turn determines motor-niche complexity. Thus, the correlation between motor complexity and brain size in primates could reflect the neural correlates of practice itself or of the sensory motor processes involved in motor skills. At present, we cannot disentangle these possibilities, but the findings imply that complexity in the motor dimension, at least in carnivorans, does not necessarily require cognitive understanding or knowledge but rather training and practice, or, in other words, time. Both life history pathways (extended provisioning and slow development) provide immature animals of both taxa with extensive practice time (see Part I of Discussion). 
Also in agreement with our prediction, the analyses in primates provided strong evidence that large brains are associated with a higher complexity in the knowledge dimension of the niche, even after controlling for the effects of the motor niche. Moreover, in figure 5, which plots knowledge-niche complexity versus relative brain size, all species cluster in the lower-right corner. This shows that, although large-brained species may live in either simple or complex knowledge niches, small-brained species are never found in niches with high knowledge complexity. This finding suggests that living in a foraging niche with high knowledge complexity is indeed cognitively challenging for a primate. These findings build on work by Barton (2012) and Reader and Laland (2002), who have found that larger brains in primates are associated with enhanced foraging skills and higher frequency of innovation and social learning, usually in the ecological domain. Overall, therefore, it is the cumulative effect of different cognitively challenging elements of a species's niche that underlies the correlation with relative brain size, and thus, cognitive abilities. In fact, the models for the separate elements of the knowledge-niche complexity score do not show strong links with brain size. The effects of brain size disappear as soon as we control for the motor component, except for tool use (see SOM for the different models). Together with the findings from Part I, this implies that neither a diverse diet nor extractive foraging alone is cognitively so challenging that it requires enlarged brain size. However, the combination of all these knowledge elements together does require enhanced cognition in primates.

The coevolution between niche complexity, brain size and developmental slowdown in primates is consistent with the needing-to-learn hypothesis, which states that the age of first reproduction is determined by the number and complexity of skills that have to be learned for adult success (Janson and van Schaik, 1993). However, these findings are also consistent with the idea that larger brains are inevitably connected to extended periods of growth and maturation, due to the developmental costs imposed by growing and maturing a large brain (Barton and Capellini, 2011). The two ideas are related. The slowdown caused by energy tradeoffs creates time to learn more complex skills.

In contrast to primates, brain size has no effect at all on knowledge foraging-niche complexity in carnivorans, neither for the separate a priori defined elements nor for the additive knowledge-niche complexity score. In other words, carnivorans do not need enhanced cognitive abilities to master these elements of their foraging niche, even though it was found that in carnivorans, diet type is correlated with brain size (Swanson et al., 2012). Together with the fact that most carnivoran species are provisioned as immature animals and the finding that higher knowledge-niche complexity is indeed associated with extended periods of provisioning, this result suggests that, in contrast to primates, successful hunting and foraging in carnivorans is built on intensive practice rather than increased knowledge. Whereas in primates, a slow, conservative development provides not only time to learn but also allows for the development of a large brain and therefore enhanced cognition, carnivorans use a more canalized pathway where provisioning buffers them from the consequences of their own incompetence and so allows them to practice specific skills until they are mastered.

\section{Implications for general mammalian and hominin evolution}

We have shown that primates and carnivorans use distinct pathways in order to meet the energetic and time requirements imposed by complex foraging niches: extended periods of provisioning in carnivorans versus a slower overall pace of development in primates. First, this pattern of correlated evolution also implies that only those primate lineages that could somehow afford to develop more slowly and only those carnivoran lineages that could afford longer post-weaning provisioning were 
able to evolve into a more complex foraging niche. Thus, each species probably reaches a foragingniche complexity where the benefits of having this more complex niche (probably especially in terms of starvation avoidance and reduced competition with other species) are balanced by the demographic costs of reduced growth rate (primates) or reproduction (carnivorans).

Our study also showed that for primates, living in a complex niche is correlated with larger brains and therefore probably requires enhanced cognitive abilities. Again, a scenario of correlated evolution is most plausible: increases in brain size allow species to exploit more complex niches (and therefore qualitatively better resources), which will in the end provide them with the energy needed to support further increases in brain size. The absence of provisioning in the vast majority of all primate species, but the presence of systematic pre- and post-weaning provisioning in many carnivoran species is most likely based on a systematic difference in the feeding biology of the two taxa. Most carnivorans are dependent on food items that are large and difficult to process but energy-rich. Thus, from an energetic perspective, provisioning of food is more effective for them compared to primates, which rely on smaller food items that are generally more easily acquired, albeit not necessarily processed. The observed differences between the two taxa, therefore, seems to be an example of constrained evolution where preexisting life history characteristics predispose species to evolve into certain directions (McKitrick, 1993; Futuyma, 1998).

Across mammals, based on our findings, we predict that opportunities for provisioning or opportunities for low mortality act as limiting factors for the possible evolution of complex niches. We therefore expect that species with high extrinsic mortality (e.g. due to high predation risk) that at the same time rely on resources that cannot be shared will be prevented from evolving into complex niches.

When applied to human hunter-gatherers, our framework might not only explain the general pattern observed across mammals but also the extreme case of the evolution of human life history and intelligence. Human hunter-gatherers occupy by far the most complex motor and knowledge niche of all mammals. Thus, hunter-gatherers have a very broad diet composed of food items that require special knowledge to acquire and intensive processing: they rely heavily on extractive foraging, tool use, and cooperative hunting (Kaplan et al., 2000). Accordingly, modern foragers reach adult-level proficiency for the more skill-intensive elements of the diet strikingly late in development and only after years of practice (Kaplan et al., 2000; Gurven et al., 2006).

Obviously, when a lineage shares food and has slow development, evolving into a knowledgecomplex niche is more likely. Thus, the most likely explanation for the evolution of a foraging-niche complexity far beyond the range of other mammals is that our ancestors, uniquely, combined very slow development and systematic provisioning of immature animals and even adults. With the adoption of systematic hunting, beginning approximately 2.5 million years ago (Dominguez-Rodrigo, 2010; Ferraro et al., 2013), our hominin ancestors began to combine a slow primate life history with a carnivoran-like niche. Meat consumption is thought to have provided the necessary energy surplus for the drastic brain size increase and coincides with enhanced technology and social adaptations such as increased levels of cooperation (Milton, 1999; Isler and van Schaik, 2012; Ferraro et al., 2013). As big game hunters, early hominins relied on big, energy rich but difficult to process food items which, as in the carnivoran lineage, most likely favored the evolution of prolonged periods of provisioning. This extended provisioning, together with a cooperative breeding system, then provided immature animals with additional time and opportunities to learn ever more complex 
ecological skills, which allowed the evolution of the unique, technologically driven human niche (Schuppli et al., 2012).

Our study provides support for the view that an ecological challenge, the complexity of the foraging niche, has shaped the life history and intelligence of the primate lineage. It thus supports the broader hypothesis that environmental factors have played a crucial role in the evolution of life history and intelligence. We did not test social effects on brain size evolution in this study but it is very likely that some of the remaining variance in our data can be explained by social variables, such as group size, as proposed by the social brain hypothesis (Byrne and Whiten, 1988; Dunbar, 1998; Barton, 2012). This general framework is in line with theories suggesting that the coevolution between complex foraging techniques and energetic needs in an increasingly seasonal environment was a crucial element during the evolution of human intelligence (Parker and Gibson, 1977; Byrne, 1997).

\section{Acknowledgements}

We thank Sandra A. Heldstab for helping to collect data on brain size and we are grateful to the following museums for allowing us to measure brain volumes of their specimens: The Field Museum Chicago, Smithsonian USNM Washington and American Museum of Natural History New York. We acknowledge the AnthroTree Workshop organized by Charlie Nunn for training in phylogenetic comparative methods. Furthermore, we are thankful to the reviewers including Rob Barton who helped to improve the manuscript. This study was funded through Swiss National Science Foundation grant No. 31003A-144210, No. 31003A-138368/1 and No. 310030B-160363/1, as well as the A.H. Schultz Foundation and the University of Zurich.

\section{References:}

Anton, S.C., Potts, R., Aiello, L.C., 2014. Evolution of early Homo: An integrated biological perspective. Science 345, 1236828.

Barrickman, N.L., Bastian, M.L., Isler, K., van Schaik, C.P., 2008. Life history costs and benefits of encephalization: a comparative test using data from long-term studies of primates in the wild. J. Hum. Evol. 54, 568-590.

Barton, R.A., 2012. Embodied cognitive evolution and the cerebellum. Phil. Trans. R. Soc. 367, 20972107.

Barton, R.A., Capellini, I., 2011. Maternal investment, life histories, and the costs of brain growth in mammals. Proc. Natl. Acad. Sci. 108, 6169-6174.

Berbesque, J.C., Marlowe, F.W., Shaw, P., Thompson, P., 2014. Hunter-gatherers have less famine than agriculturalists. Biol. Lett. 10, 20130853.

Bininda-Emonds, O.R.P., Cardillo, M., Jones, K.E., MacPhee, R.D.E., Beck, R.M.D., Grenyer, R., Price, S.A., Vos, R.A., Gittleman, J.L., Purvis, A., 2007. The delayed rise of present-day mammals. Nature 446, 507-512.

Boesch, C., 2002. Cooperative hunting roles among Tai chimpanzees. Hum. Nat. 13, 27-46.

Byrne, R.W., 1997. The technical intelligence hypothesis: an additional evolutionary stimulus to intelligence? In: Whiten, A., Byrne, R.W. (Eds.), Machiavellian intelligence II: extensions and evaluations. Cambridge University Press, Cambridge, pp. 289-311.

Byrne, R.W., Whiten, A., Henzi, S.P., McCulloch, F.M., 1993. Nutritional constraints on mountain baboons (Papio Ursinus) - Implications for baboon socioecology. Behav. Ecol. Sociobiol. 33, 233-246.

Deaner, R.O., Isler, K., Burkart, J., van Schaik, C., 2007. Overall brain size, and not encephalization quotient, best predicts cognitive ability across non-human primates. Brain Behav. Evol. 70, 115-124.

Dittus, W.P.J., 1977. Social regulation of population density and age- sex distributions in toque monkey. Behaviour 63, 281-322. 
Dominguez-Rodrigo, M., Pickering, T.R., Bunn, H.T., 2010. Configurational approach to identifying the earliest hominin butchers. Proc. Natl. Acad. Sci. 107, 20929-20934.

Dunbar, R.I.M., 1998.The social brain hypothesis. Evol. Anthropol. 6, 178-190.

Ferraro, J.V., Braun, D.R., Hertel, F., Potts, R., Pobiner, B.L., Oliver, J.S., Seaman, J.W., III, Binetti, K.M., Seaman, J.W., Jr., Plummer, T.W., Ditchfield, P.W., Bishop, L.C., 2013. Earliest Archaeological Evidence of Persistent Hominin Carnivory. PLoS ONE 8, e62174. doi:10.1371/journal.pone.0062174 Fish, J.L.

Lockwood, C.A., 2003. Dietary constraints on encephalization in primates. Am. J. Phys. Anthropol. $120,171-181$.

Futuyma, D.J., 1998. Evolutionary Biology. 3rd ed. Sinauer Associates.

Gibson, K.R., 1986. Cognition, brain size and the extraction of embedded food resources. In: Else, J.G., Lee P.C. (Eds.), Primate Ontogeny, Cognition and Social Behaviour. Cambridge University Press, Cambridge, pp. 93-103.

Gonzalez-Lagos, C., Sol, D., Reader, S.M., 2010. Large-brained mammals live longer. Evol. Biol. 23, 1064-1074.

Gunst, N., Boinski, S., Fragaszy, D.M., 2008. Acquisition of foraging competence in wild brown capuchins (Cebus apella), with special reference to conspecifics' foraging artefacts as an indirect social influence. Behaviour 145, 195-229.

Gunst, N., Leca, J.-B., Boinski, S., Fragaszy, D., 2010. The Ontogeny of Handling Hard-to-Process Food in Wild Brown Capuchins (Cebus apella apella): Evidence from Foraging on the Fruit of Maximiliana maripa. Am. J. Primatol. 72, 960-973.

Gurven, M., Kaplan, H., Gutierrez, M., 2006. How long does it take to become a proficient hunter? Implications for the evolution of extended development and long life span. J. Hum. Evol. 51, 454-470.

Hanya, G., 2003. Age differences in food intake and dietary selection of wild male Japanese macaques. Primates 44, 333-339.

Harvey, P.H., Cluttonbrock, T.H., 1985. Life-history variation in primates. Evolution 39, 559-581.

Ho, L.S.T., Ane, C., 2014. The phylolm package: Phylogenetic Linear Regression. Syst. Biol. 63, 397408.

Holekamp, K.E., Smale, L., Berg, R., Cooper, S.M., 1997. Hunting rates and hunting success in the spotted hyena (Crocuta crocuta). J. Zool. 242, 1-15.

Isler, K., van Schaik, C.P., 2009. The Expensive Brain: A framework for explaining evolutionary changes in brain size. J. Hum. Evol. 57, 392-400.

Isler, K., van Schaik, C.P., 2012. Allomaternal care, life history and brain size evolution in mammals. J. Hum. Evol. 63, 52-63.

Ives, A.R., Garland, T., 2010. Phylogenetic logistic regression for binary dependent variables. Syst. Biol. 59, 9-26.

Janson, C.H., van Schaik, C.P., 1993. Ecological risk aversion in juvenile primates: slow and steady wins the race. In: Pereira, M.E., Fairbanks, L.A. (Eds.), Juvenile Primates: Life History, Development, and Behavior. The University of Chicago Press, Chicago and London, pp. 57-76.

Johnson, S.E., Bock, J., 2004. Trade-offs in skill acquisition and time allocation among juvenile chacma baboons. Hum. Nat. 15, 45-62.

Kaplan, H., Hill, K., Lancaster, J., Hurtado, A.M., 2000. A theory of human life history evolution: Diet, intelligence, and longevity. Evol. Anthropol. 9, 156-185.

Kuzawa, C.W., Chugani, H.T., Grossman, L.I., Lipovich, L., Muzik, O., Hof, P.R., Wildman, D.E., Sherwood, C.C., Leonard, W.R., Lange, N., 2014. Metabolic costs and evolutionary implications of human brain development. Proc. Natl. Acad. Sci. 111, 13010-13015.

Lefebvre, L., Reader, S.M., Sol, D., 2004. Brains, innovations and evolution in birds and primates. Brain Behav. Evol. 63, 233-246.

Leonard, W.R., Robertson, M.L., 1997. Comparative primate energetics and hominid evolution. Am. J. Phys. Anthropol. 102, 265-281.

Lonsdorf, E.V., 2006. What is the role of mothers in the acquisition of termite-fishing behaviors in wild chimpanzees (Pan troglodytes schweinfurthii)? Anim. Cognit. 9, 36-46. 
Matthews, L.J., Arnold, C., Machanda, Z., Nunn, C.L., 2010. Primate extinction risk and historical patterns of speciation and extinction in relation to body mass. Phil. Trans. R. Soc. DOI: $10.1098 / \mathrm{rspb} .2010 .1489$.

McKitrick, M.C., 1993. Phylogenetic constraint in evolutionary theory: has it any explanatory power? A. Rev. Ecol. Syst. 24, 307-330.

Meulman, E.J.M., van Schaik, C.P., 2013. Orangutan tool use and the evolution of technology. In: Sanz, C.M., Call, J., Boesch Ch. (Eds.), Tool Use in Animals: Cognition and Ecology. Cambridge University Press, Cambridge, pp. 176-202.

Milton, K., 1981. Distribution patterns of tropical plant foods as an evolutionary stimulus to primate mental development. Am. Anthropol. 83, 534-548.

Milton, K., 1988. Foraging behaviour and the evolution of primate intelligence. In: Byrne, R., Whiten A. (Eds.), Machiavellian intelligence: social expertise and the evolution of intellect in monkeys, apes and humans. Clarendon Press, Oxford, pp. 285-308.

Milton, K., 1999. A hypothesis to explain the role of meat-eating in human evolution. Evol. Anthropol. 8, 11-21.

Myers, P., Espinosa, R., Parr, C.S., Jones, T., Hammond, G.S., Dewey, T.A., 1995. The Animal Diversity Web (online). University of Michigan. http://animaldiversity.org.

Nakayama, Y., Matsuoka, S., Watanuki, Y., 1999. Feeding rates and energy deficits of juvenile and adult Japanese monkeys in a cool temperate area with snow coverage. Ecol. Res. 14, 291301.

Nowak, R.M., Paradiso, J.L., Nowak, R.M., Paradiso, J.L., 1983. Walker's mammals of the world. Volume $1 \&$ 2. The Johns Hopkins University Press, Baltimore, Maryland.

Nunn, C.L., 2011. The Comparative Approach in Evolutionary Anthropology and Biology. The University of Chicago Press, Chicago and London.

Orme, D., 2011. The caper package: comparative analysis of phylogenetics and evolution in R.

Pagel, M., 1999. The maximum likelihood approach to reconstructing ancestral character states of discrete characters on phylogenies. Syst. Biol. 48, 612-622.

Parker, S.T., Gibson, K.R., 1977. Object manipulation, tool use and sensorimotor intelligence as feeding adaptations in Cebus monkeys and great apes. J. Hum. Evol. 6, 623-641.

Perelman, P., Johnson, W.E., Roos, C., Seuanez, H.N., Horvath, J.E., Moreira, M.A.M., Kessing, B., Pontius, J., Roelke, M., Rumpler, Y., Schneider, M.P.C., Silva, A., O'Brien, S.J., Pecon-Slattery, J., 2011. A Molecular Phylogeny of Living Primates. Plos Genet. 7, e1001342.

R Core Team, 2012. R: A language and environment for statistical computing. R Foundation for Statistical Computing, Vienna.

Reader, S.M., Laland, K.N., 2002. Social intelligence, innovation, and enhanced brain size in primates. Proc. Natl. Acad. Sci. 99, 4436-4441.

Ross, C., Jones, K., 1999. Socioecology and the evolution of primate reproductive rates. In: Lee, P., (Ed.), Comparative Primate Socioecology. Cambridge University Press, pp. 73-110.

Sand, H., Wikenros, C., Wabakken, P., Liberg, O., 2006. Effects of hunting group size, snow depth and age on the success of wolves hunting moose. Anim. Behav. 72, 781-789.

Sargeant, B.L., Mann, J., Berggren, P., Krützen, M., 2005. Specialization and development of beach hunting, a rare foraging behavior, by wild bottlenose dolphins (Tursiops sp.). Can. J. Zool. 83, 1400-1410.

Schuppli, C., Isler, K., van Schaik, C.P., 2012. How to explain the unusually late age at skill competence among humans. J. Hum. Evol. 63, 843-850.

Stone, A.I., 2006. Foraging ontogeny is not linked to delayed maturation in squirrel monkeys (Saimiri sciureus). Ethology 112, 105-115.

Swanson, E.M., Holekamp, K.E., Lundrigan, B.L., Arsznov, B.M., Sakai, S.T., 2012. Multiple Determinants of Whole and Regional Brain Volume among Terrestrial Carnivorans. PLoS One 7, e38447-e38447.

Van Woerden, J.T., van Schaik, C.P., Isler, K., 2010. Effects of seasonality on brain size evolution: evidence from strepsirrhine primates. Am. Nat. 176, 758-767.

Van Woerden, J.T., Willems, E.P., van Schaik, C.P., Isler, K., 2012. Large brains buffer energetic effects 
of seasonal habitats in catarrhine primates. Evolution 66, 191-199.

Van Woerden, J.T., van Schaik, C.P., Isler, K., 2014. Seasonality of diet composition is related to brain size in New World Monkeys. Am. J. Phys. Anthropol. 154, 628-632.

Walker, R., Burger, O., Wagner, J., Von Rueden, C.R., 2006. Evolution of brain size and juvenile periods in primates. J. Hum. Evol. 51, 480-489.

Watt, J., 1993. Ontogeny of hunting behavior of otters (Lutra Lutra) in a marine environment. In: Dunstone, N., Gorman, M.L. (Eds.), Mammals as Predators. Oxford University Press, pp. 87104.

\section{Figures}

Figure 1. Slow development and extended provisioning have been shown to allow for extended periods of learning (later relative age at skill competence [Schuppli at al., 2012]). Here we ask in Part I whether the same two factors ultimately allow species to evolve into more complex niches. In Part II we are interested in how niche complexity relates to relative brain size and expect only the knowledge niche, but not necessarily the motor niche, component to be associated with large relative brain size.

Figure 2. Relationships between pace of development (age at first reproduction corrected for body mass) and motor-niche complexity for primates and between length of provisioning (corrected for body mass) and motorniche complexity for carnivorans.

Figure 3. Relationships between pace of development (age at first reproduction corrected for body mass) or provisioning (corrected for body mass) and the additive knowledge-niche complexity score in primates and carnivorans.

Figure 4. Relationships between relative brain size (corrected for body mass) and motor-niche complexity for primates and carnivorans.

Figure 5. Relationships between relative brain size (corrected for body mass) and additive knowledge-niche complexity score for primates and carnivorans. 
Table 1. Motor-niche complexity categories.

\begin{tabular}{ll} 
Motor-niche complexity & Niche \\
\hline \hline 1 & folivory and grazing \\
2 & frugivory and insectivory \\
3 & extractive foraging and mobile prey catching \\
\hline \hline
\end{tabular}


Table 2. Distribution of the different knowledge-niche complexity eleme additive knowledge-niche complexity score in primates and carnivorans.

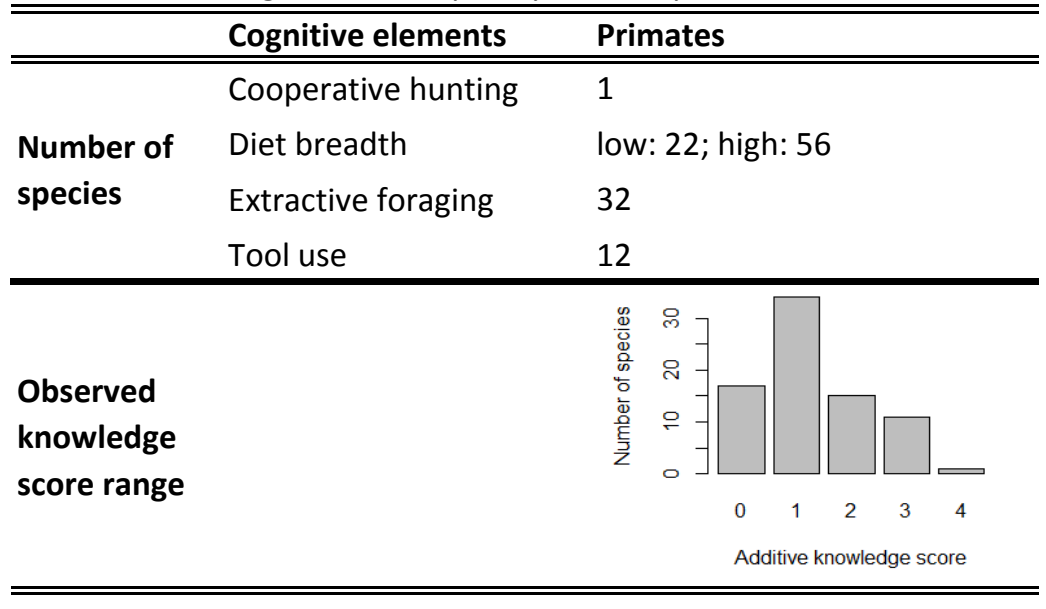


Table 3. Phylogenetic least-square multiple regression models with motor-niche complexity as the response variable and body mass, AFR and length of provisioning as the predictor variables, analyzed separately for the a) 78 primate and b) 65 carnivoran species. Statistical significance $(p<0.05)$ is indicated by bold font.

a)

\begin{tabular}{|c|c|c|c|c|c|c|}
\hline \multicolumn{7}{|l|}{ Primates $n=78$} \\
\hline dependent variable & $\mathbf{R}^{2}$ & $\begin{array}{c}\text { phylogenetic } \\
\text { signal }\end{array}$ & predictor variables & estimate & std. error & $p$-value \\
\hline \multirow{3}{*}{$\begin{array}{c}\text { motor-niche } \\
\text { complexity }\end{array}$} & \multirow{3}{*}{0.22} & \multirow{3}{*}{$\lambda=0.87$} & $\log$ body & -0.334 & 0.104 & 0.002 \\
\hline & & & $\log A F R$ & 0.988 & 0.236 & $<0.001$ \\
\hline & & & log provisioning & -0.012 & 0.139 & 0.930 \\
\hline \multicolumn{7}{|l|}{ b) } \\
\hline \multicolumn{7}{|l|}{ Carnivorans $n=65$} \\
\hline dependent variable & $\mathbf{R}^{2}$ & $\begin{array}{c}\text { phylogenetic } \\
\text { signal }\end{array}$ & predictor variables & estimate & std. error & $p$-value \\
\hline \multirow{3}{*}{$\begin{array}{l}\text { motor-niche } \\
\text { complexity }\end{array}$} & \multirow{3}{*}{0.16} & \multirow{3}{*}{$\lambda=0.78$} & log body & -0.123 & 0.088 & 0.168 \\
\hline & & & $\log A F R$ & -0.228 & 0.203 & 0.264 \\
\hline & & & log provisioning & 0.553 & 0.173 & 0.002 \\
\hline
\end{tabular}


Table 4. Phylogenetic least-square multiple regression models with the additive knowledge-niche complexity score as response variable and body mass, AFR and length of provisioning as predictor variables analyzed separately for the a) 78 primate and b) 65 carnivoran species. Statistical significance $(p<0.05)$ is indicated by bold font.

a)

Primates $n=78$

\begin{tabular}{cccrccc} 
dependent variable & $\mathbf{R}^{\mathbf{2}}$ & $\begin{array}{c}\text { phylogenetic } \\
\text { signal }\end{array}$ & predictor variables & estimate & std. error & $\boldsymbol{p}$-value \\
\hline \hline \multirow{2}{*}{$\begin{array}{c}\text { knowledge-niche } \\
\text { complexity }\end{array}$} & \multirow{2}{*}{0.41} & \multirow{2}{*}{$\lambda=0.00$} & $\log$ body & -0.767 & 0.308 & $\mathbf{0 . 0 1 5}$ \\
& & & $\log$ AFR & 3.701 & 0.764 & $<0.001$ \\
& & log provisioning & 0.122 & 0.478 & 0.800 \\
\hline \hline
\end{tabular}

b)

\begin{tabular}{cccrccc}
\hline \hline Carnivorans $n=65$ & & & & & \\
dependent variable & $\mathbf{R}^{2}$ & $\begin{array}{c}\text { phylogenetic } \\
\text { signal }\end{array}$ & predictor variables & estimate & std. error & $\boldsymbol{p}$-value \\
\hline \hline & & & $\log$ body & -0.080 & 0.125 & 0.526 \\
$\begin{array}{c}\text { knowledge-niche } \\
\text { complexity }\end{array}$ & 0.15 & $\lambda=0.25$ & $\log$ AFR & -0.452 & 0.284 & 0.118 \\
& & & log provisioning & 0.790 & 0.284 & $\mathbf{0 . 0 0 7}$ \\
\hline \hline
\end{tabular}


Table 5. Phylogenetic least-square multiple regression models with motor-niche complexity as the response variable and body mass and brain size as the predictor variables, analyzed separately for the a) 78 primate and b) 65 carnivoran species. Statistical significance $(p<0.05)$ is indicated by bold font.

a)

\begin{tabular}{|c|c|c|c|c|c|c|}
\hline $\begin{array}{l}\text { Primates } n=78 \\
\text { dependent variable }\end{array}$ & $\mathbf{R}^{2}$ & $\begin{array}{c}\text { phylogenetic } \\
\text { signal }\end{array}$ & predictor variables & estimate & std. error & $p$-value \\
\hline $\begin{array}{l}\text { motor-niche } \\
\text { complexity }\end{array}$ & 0.12 & $\lambda=0.66$ & $\begin{array}{l}\text { log body } \\
\text { log brain }\end{array}$ & $\begin{array}{l}-0.715 \\
0.880\end{array}$ & $\begin{array}{l}0.224 \\
0.294\end{array}$ & $\begin{array}{l}0.002 \\
0.004\end{array}$ \\
\hline b) & & & & & & \\
\hline $\begin{array}{l}\text { Carnivorans } n=65 \\
\text { dependent variable }\end{array}$ & $\mathbf{R}^{2}$ & $\begin{array}{c}\text { phylogenetic } \\
\text { signal }\end{array}$ & predictor variables & estimate & std. error & $p$-value \\
\hline $\begin{array}{l}\text { motor-niche } \\
\text { complexity }\end{array}$ & 0.00 & $\lambda=0.87$ & $\begin{array}{l}\text { log body } \\
\text { log brain }\end{array}$ & $\begin{array}{l}0.051 \\
-0.080\end{array}$ & $\begin{array}{l}0.169 \\
0.264\end{array}$ & $\begin{array}{l}0.761 \\
0.764\end{array}$ \\
\hline
\end{tabular}


Table 6. Phylogenetic least-square multiple regression models with the additive knowledge-niche complexity score as response variable and body mass and brain size as predictor variables analyzed separately for the a) 78 primate and b) 65 carnivoran species. Statistical significance $(p<0.05)$ is indicated by bold font.

a)

Primates $n=78$

\begin{tabular}{ccccccc} 
dependent variable & $\mathbf{R}^{2}$ & $\begin{array}{c}\text { phylogenetic } \\
\text { signal }\end{array}$ & predictor variables & estimate & std. error & $\boldsymbol{p}$-value \\
\hline \hline $\begin{array}{c}\text { knowledge-niche } \\
\text { complexity }\end{array}$ & 0.41 & $\lambda=0.00$ & log body & -2.196 & 0.538 & $<0.001$ \\
& & & log brain & 3.600 & 0.654 & $<0.001$ \\
\hline \hline
\end{tabular}

b)

Carnivorans $n=65$

dependent variable $R^{2}$ hylogenetic signi predictor variables estimate std. error $p$-value

\begin{tabular}{ccccccc}
\hline \hline $\begin{array}{c}\text { knowledge-niche } \\
\text { complexity }\end{array}$ & 0.02 & $\lambda=0.17$ & log body & 0.185 & 0.254 & 0.471 \\
\hline \hline
\end{tabular}




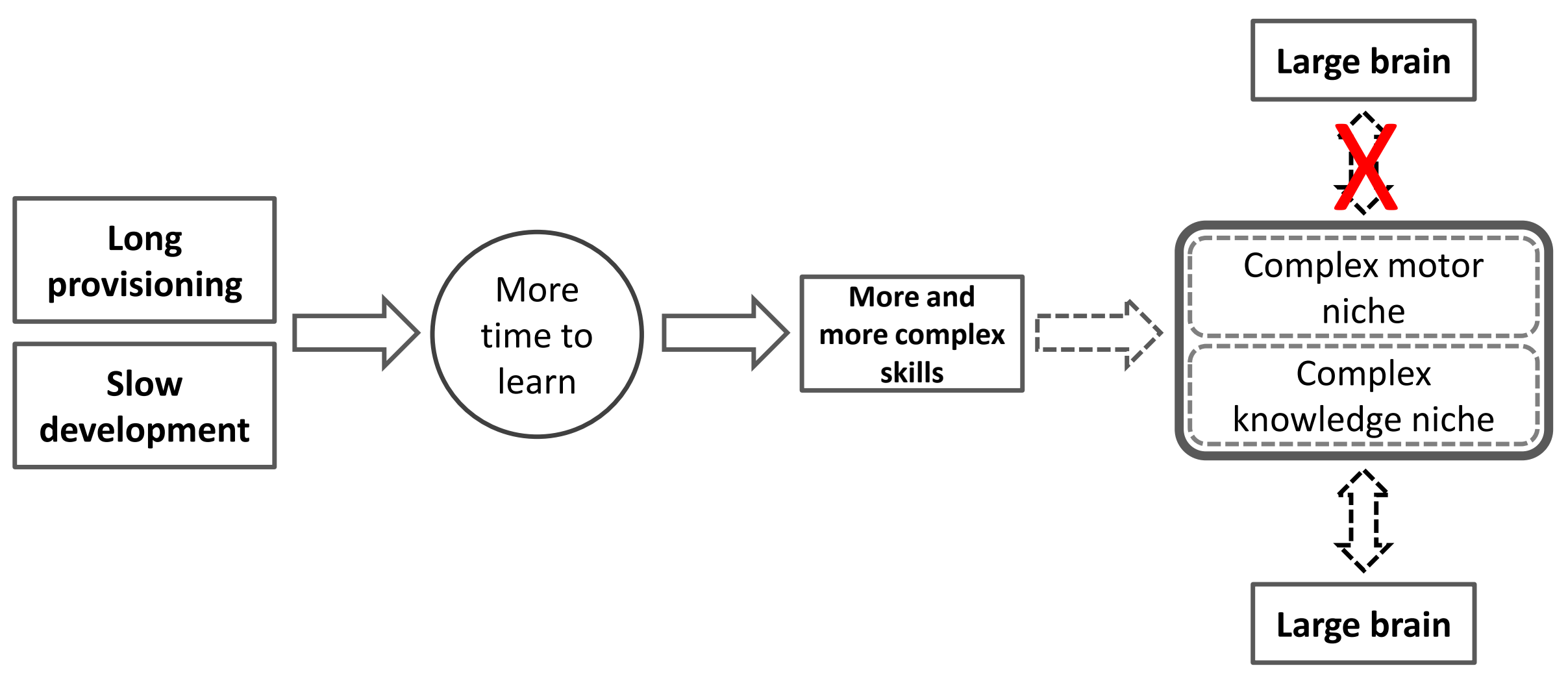


Primates

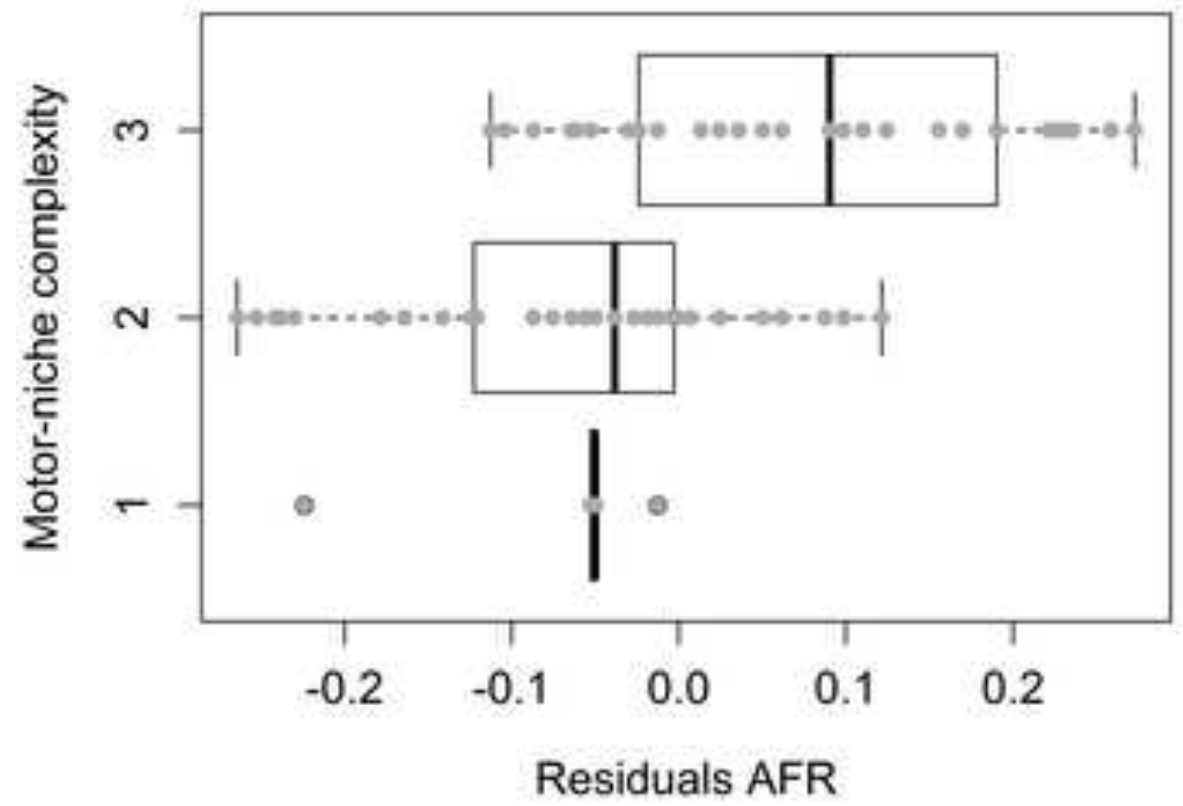

Carnivorans

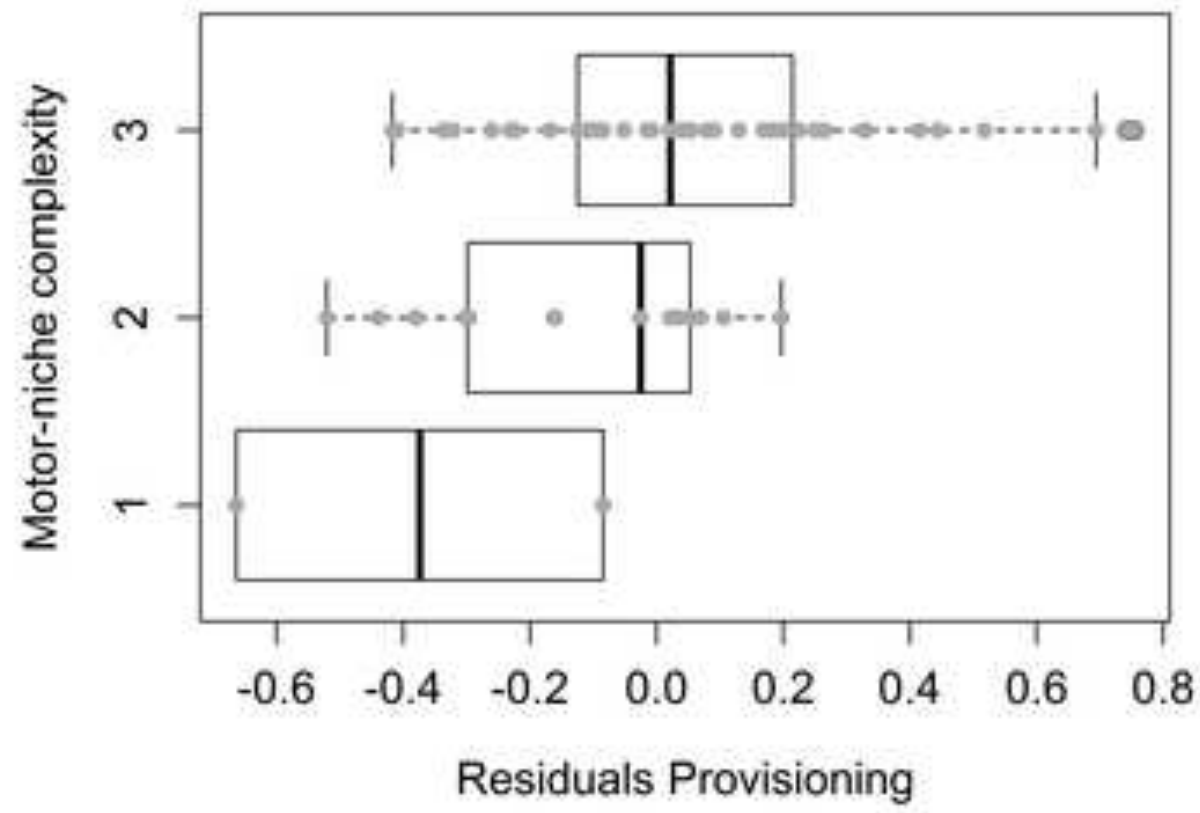


Primates

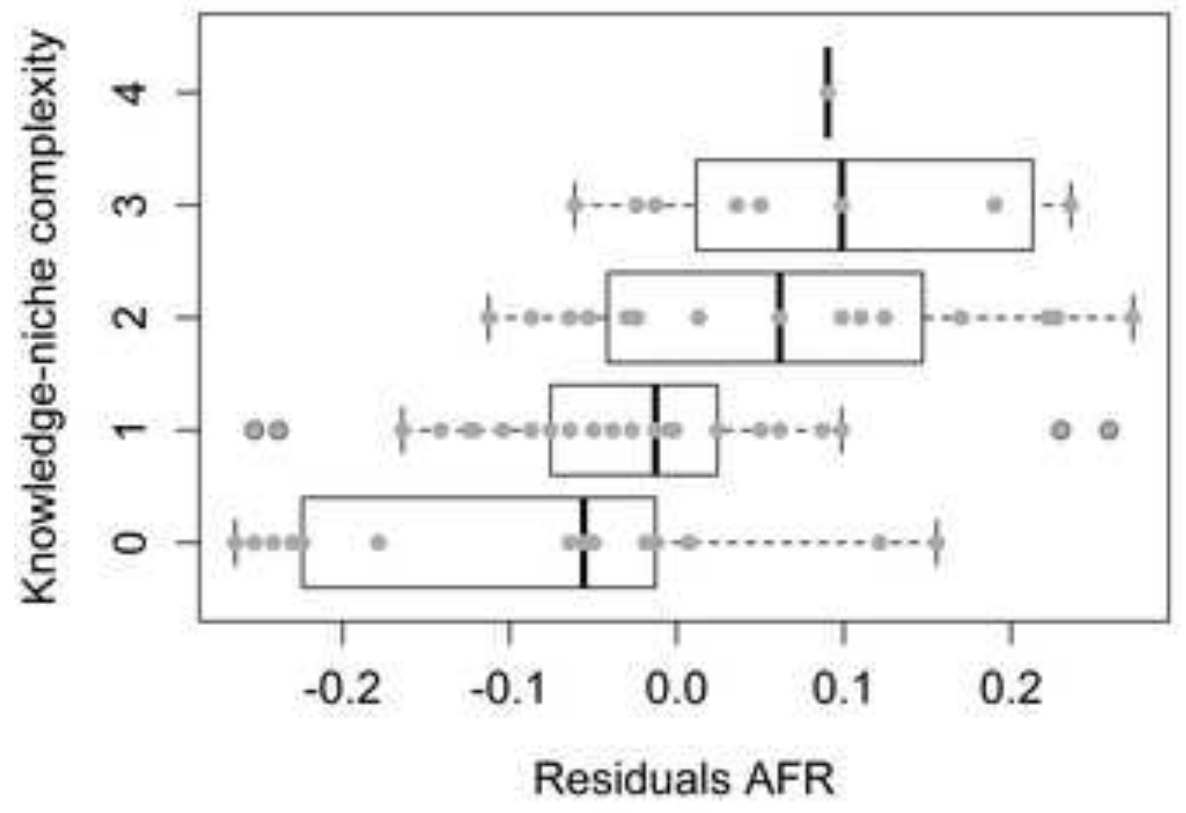

Carnivorans

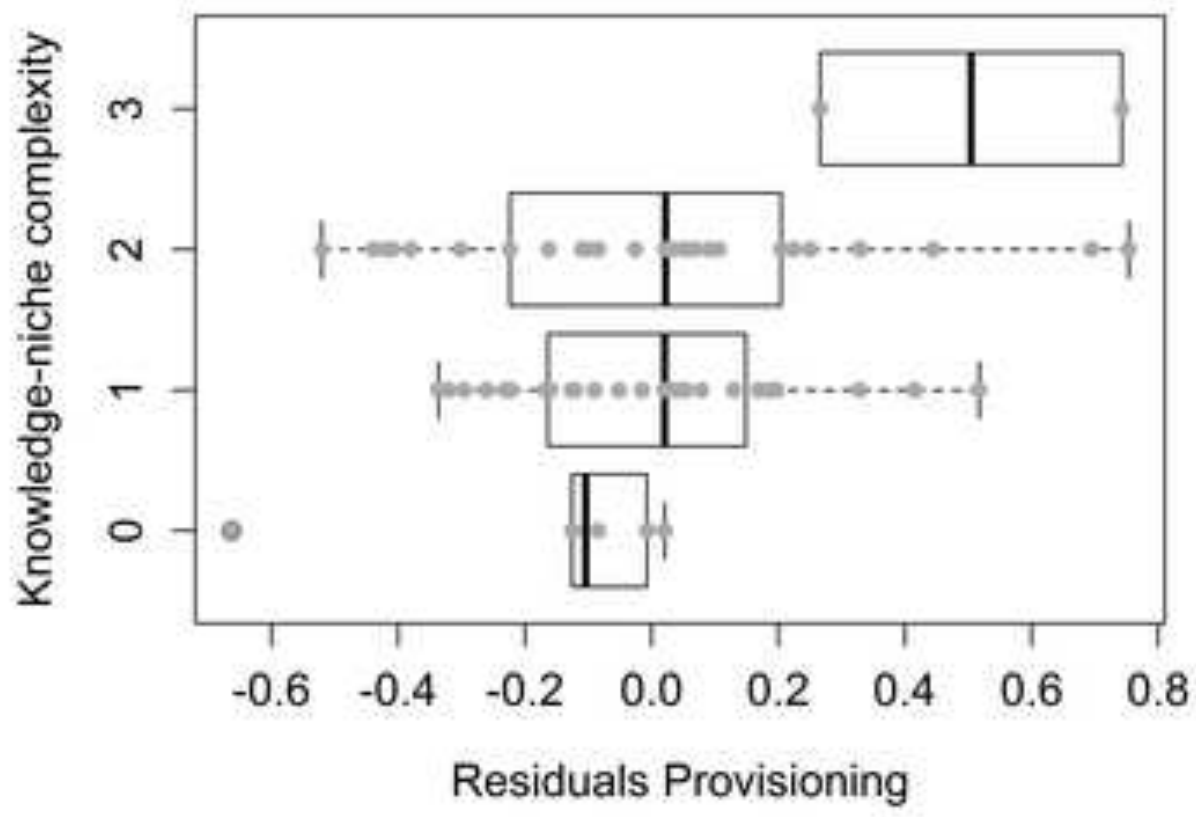


Primates

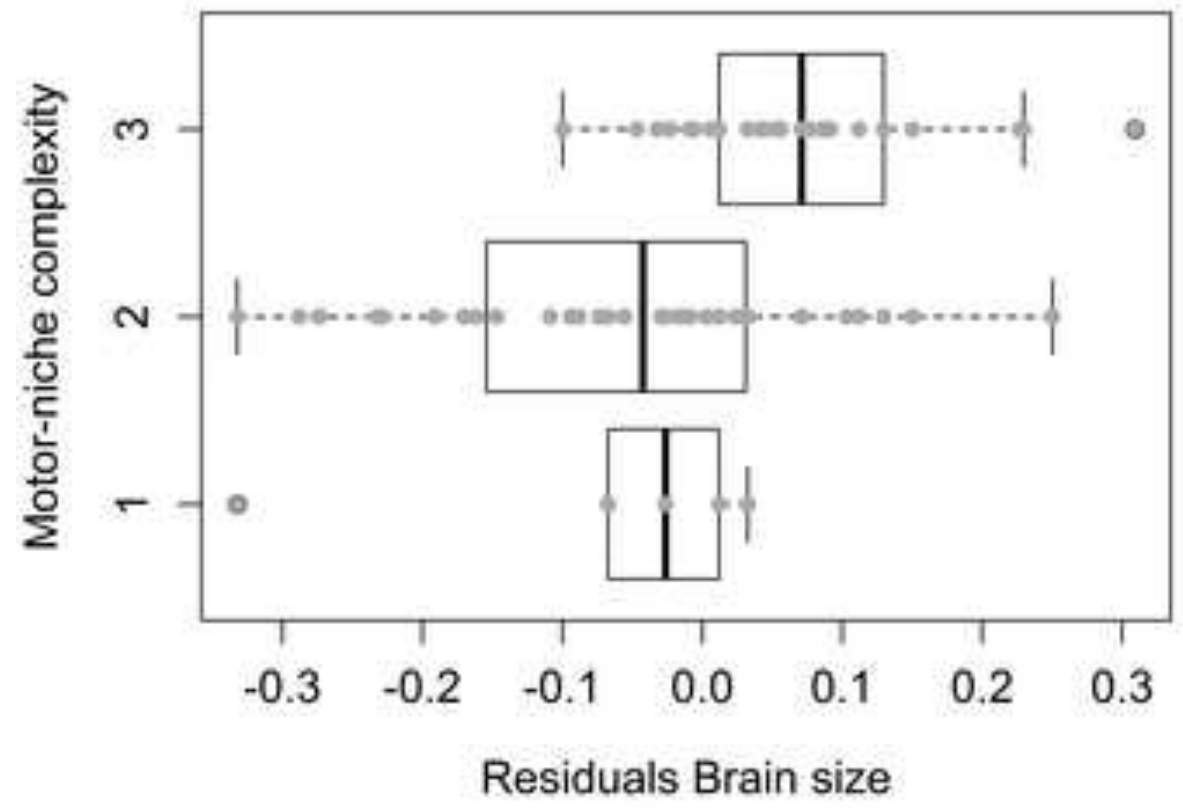

Carnivorans

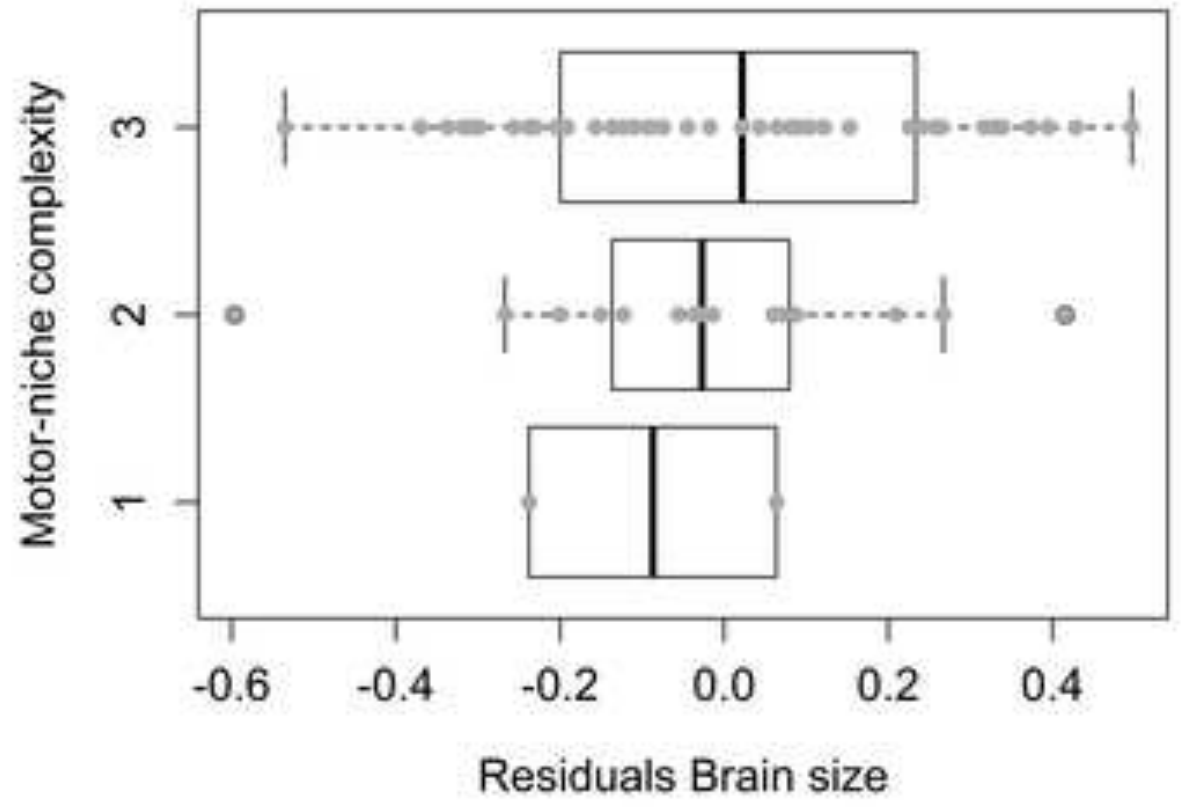


Primates

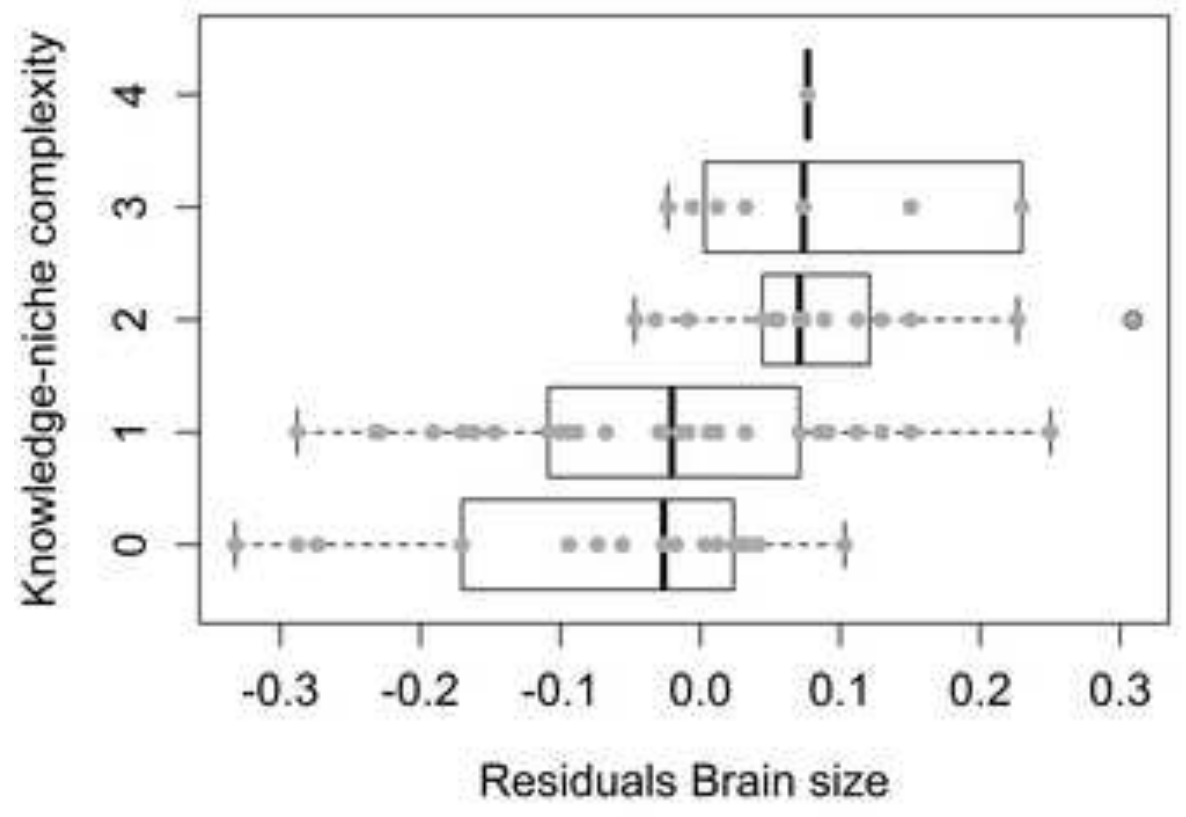

Carnivorans

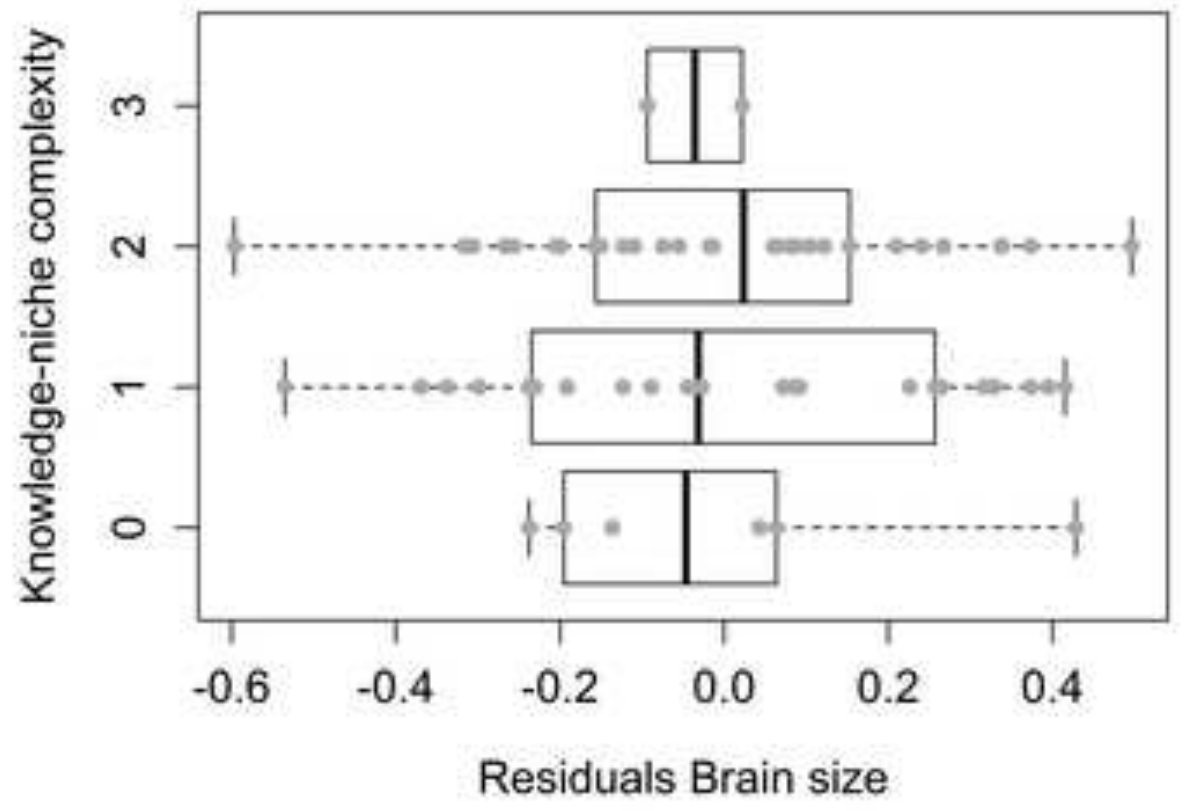

